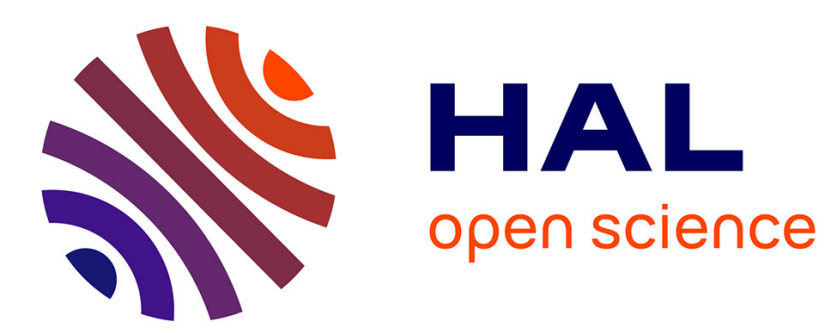

\title{
On the Behavior of Dempster's Rule of Combination and the Foundations of Dempster-Shafer Theory
}

\author{
Albena Tchamova, Jean Dezert
}

\section{To cite this version:}

Albena Tchamova, Jean Dezert. On the Behavior of Dempster's Rule of Combination and the Foundations of Dempster-Shafer Theory. International Conference Intelligent Systems, Sep 2012, Sofia, Bulgaria. 6 p. hal-00732933

\section{HAL Id: hal-00732933 \\ https://hal.science/hal-00732933}

Submitted on 17 Sep 2012

HAL is a multi-disciplinary open access archive for the deposit and dissemination of scientific research documents, whether they are published or not. The documents may come from teaching and research institutions in France or abroad, or from public or private research centers.
L'archive ouverte pluridisciplinaire HAL, est destinée au dépôt et à la diffusion de documents scientifiques de niveau recherche, publiés ou non, émanant des établissements d'enseignement et de recherche français ou étrangers, des laboratoires publics ou privés. 


\title{
On the Behavior of Dempster's Rule of Combination and the Foundations of Dempster-Shafer Theory
}

\author{
Albena Tchamova ${ }^{1}$, Jean Dezert ${ }^{2}$ \\ 1. Institute for Information and Communication Technologies, Bulgarian Academy of Sciences, \\ "Acad.G.Bonchev" Str., bl.25A, 1113 Sofia, Bulgaria. \\ Email: tchamova@bas.bg \\ 2. ONERA - The French Aerospace Lab, F-91761 Palaiseau, France. \\ Email: jean.dezert@onera.fr
}

\begin{abstract}
On the base of simple emblematic example we analyze and explain the inconsistent and inadequate behavior of Dempster-Shafer's rule of combination as a valid method to combine sources of evidences. We identify the cause and the effect of the dictatorial power behavior of this rule and of its impossibility to manage the conflicts between the sources. For a comparison purpose, we present the respective solution obtained by the more efficient PCR5 fusion rule proposed originally in Dezert-Smarandache Theory framework. Finally, we identify and prove the inherent contradiction of Dempster-Shafer Theory foundations.
\end{abstract}

Keywords-Belief functions; Dempster-Shafer Theory; DSmT; PCR5; contradiction.

\section{INTRODUCTION}

Dempster-Shafer Theory (DST), also known as the Theory of Evidence or the Theory of Belief Functions, was introduced by Shafer in 1976 [1] based on Dempster's previous works [2], [3], [4]. This theory offers an elegant theoretical framework for modeling uncertainty, and provides a method for combining distinct bodies of evidence collected from different sources. In the past more than three decades, DST has been used in many applications, in fields including information fusion, pattern recognition, and decision making [5].

In spite of it, starting from Zadeh's criticism [6], [7], [8], many questions have arisen about the validity and the consistency of this theory when combining uncertain and conflicting evidences expressed as basic belief assignments (bba's). Besides Zadeh's example, there have been several detailed analyses on this topic by Lemmer [9], Voorbraak [10] and Wang [11]. Other authors like Pearl [12] and Walley [13], and more recently Gelman [14], have also warned the "belief function community" about this fundamental problem, i.e., the validity of Dempster-Shafer's rule ${ }^{1}$ (DS rule for short) for combining distinct pieces of evidences. Since the mid1990's, many researchers and engineers working with belief functions in applications have observed and admitted that DS

\footnotetext{
${ }^{1}$ This rule is also called Dempster's rule in the literature because it was originally proposed by Dempster. We prefer to name it Dempster-Shafer's rule because it has widely been promoted by Shafer in his development of theory of belief functions (a.k.a. DST).
}

rule is problematic for evidence combination, specially when the sources of evidence are highly conflicting.

In response to this challenge, various attempts have been made to circumvent the counter-intuitive behaviors of DS rule. They either replace Dempster-Shafer's rule by alternative rules, listed for example in [15] (Vol. 1), or apply novel semantic interpretations to the functions [15], [16], [17]. This work is based on preliminary ideas presented in the Spring School on Belief Functions Theory and Applications (BFTA) in April 2011 [18], and on many fruitful discussions with colleagues using belief functions. We start from a very basic, but emblematic example to show what is really questionable in DS rule. We will demonstrate that the main problem applying DS rule comes not from the level of conflict between sources to combine, but from the underlying interpretation of evidence and degree of belief on which the combination rule is based. We will make a comparison with respective results, obtained by using Proportional Conflict Redistribution rule no.5 (PCR5) defined within Dezert-Smarandache Theory (DSmT) [15]. In Section II we briefly recall basics of DST and DS rule. Basics of PCR5 fusion rule are outlined in Section III. In Section IV we describe our basic example and discuss the counterintuitive result obtained by DS rule and its strange behavior corresponding to the dictatorial power of particular source of evidence with respect to all another sources. A comparison with respective results obtained by PCR5 fusion rule is also made. After a discussion on dictatorial power of DempsterShafer's rule in Section V, we establish and prove in Section VI a fundamental theorem on the contradiction, grounded in DST foundations. Our concluding remarks will be given in Section VII.

\section{BASICS OF DST}

Let $\Theta=\left\{\theta_{1}, \theta_{2}, \ldots, \theta_{n}\right\}$ be a frame of discernment of a problem under consideration containing $n$ distinct elements $\theta_{i}, i=1, \ldots, n$. A basic belief assignment (bba, also called a belief mass function) $m():. 2^{\Theta} \rightarrow[0,1]$ is a mapping from the power set of $\Theta$ (i.e. the set of subsets of $\Theta$ ), denoted $2^{\Theta}$, to $[0,1]$, that must satisfy the following conditions: 1) $m(\emptyset)=$ 
0 , i.e. the mass of empty set (impossible event) is zero; 2) $\sum_{X \in 2^{\Theta}} m(X)=1$, i.e. the mass of belief is normalized to one. $m(X)$ represents the mass of belief exactly committed to $X$. An element $X \in 2^{\Theta}$ is called a focal element if and only if $m(X)>0$. The set $\mathcal{F}(m) \triangleq\left\{X \in 2^{\Theta} \mid m(X)>0\right\}$ of all focal elements of a bba $m($.$) is called the core of the$ bba. By definition, a Bayesian bba $m($.$) is a bba having only$ focal elements of cardinality 1 . The vacuous bba characterizing full ignorance is defined by $m_{v}():. 2^{\Theta} \rightarrow[0 ; 1]$ such that $m_{v}(X)=0$ if $X \neq \Theta$, and $m_{v}(\Theta)=1$.

From any bba $m($.$) , the belief function \operatorname{Bel}($.$) and the plau-$ sibility function $P l($.$) are defined as \forall X \in 2^{\Theta}: \operatorname{Bel}(X)=$ $\sum_{Y \mid Y \subseteq X} m(Y)$ and $P l(X)=\sum_{Y \mid X \cap Y \neq \emptyset} m(Y) \cdot \operatorname{Bel}(X)$ represents the whole mass of belief that comes from all subsets of $\Theta$ included in $X$. It is interpreted as the lower bound of the probability of $X$, i.e. $P_{\min }(X)$. $\operatorname{Bel}($.$) is a subadditive mea-$ sure since $\sum_{\theta_{i} \in \Theta} \operatorname{Bel}\left(\theta_{i}\right) \leq 1 . P l(X)$ represents the whole mass of belief that comes from all subsets of $\Theta$ compatible with $X$ (i.e., those intersecting $X) . P l(X)$ is interpreted as the upper bound of the probability of $X$, i.e. $P_{\max }(X) . P l($. is a superadditive measure since $\sum_{\theta_{i} \in \Theta} \operatorname{Pl}\left(\theta_{i}\right) \geq 1$. $\operatorname{Bel}(X)$ and $P l(X)$ are classically seen as lower and upper bounds of an unknown probability $P($.$) and one has the following$ inequality satisfied $\operatorname{Bel}(X) \leq P(X) \leq P l(X), \forall X \in 2^{\Theta}$.

The DS rule of combination [1] is a mathematical operation, denoted $\oplus$, which corresponds to the normalized conjunctive fusion rule. Based on Shafer's model of the frame, the combination of two independent and distinct sources of evidences characterized by their bba $m_{1}($.$) and m_{2}($.$) and related to the$ same frame of discernment $\Theta$ is defined by $m_{D S}(\emptyset)=0$, and $\forall X \in 2^{\Theta} \backslash\{\emptyset\}$ by

$$
m_{D S}(X)=\left[m_{1} \oplus m_{2}\right](X)=\frac{m_{12}(X)}{1-K_{12}}
$$

where

$$
m_{12}(X) \triangleq \sum_{\substack{X_{1}, X_{2} \in 2^{\Theta} \\ X_{1} \cap X_{2}=X}} m_{1}\left(X_{1}\right) m_{2}\left(X_{2}\right)
$$

corresponds to the conjunctive consensus on $X$ between the two sources of evidence. $K_{12}$ is the total degree of conflict between the two sources of evidence defined by

$$
K_{12} \triangleq m_{12}(\emptyset)=\sum_{\substack{X_{1}, X_{2} \in 2^{\Theta} \\ X_{1} \cap X_{2}=\emptyset}} m_{1}\left(X_{1}\right) m_{2}\left(X_{2}\right)
$$

When $K_{12}=m_{12}(\emptyset)=1$, the two sources are said to be in total conflict and their combination cannot be applied since DS rule (1) is mathematically not defined because of $0 / 0$ indeterminacy [1]. DS rule is commutative and associative which makes it very attractive from engineering implementation standpoint, since the combinations of sources can be done sequentially instead globally and the order doesn't matter. Moreover, the vacuous bba is a neutral element for the DS rule, i.e. $\left[m \oplus m_{v}\right]()=.\left[m_{v} \oplus m\right]()=.m($.$) for any bba m($. defined on $2^{\Theta}$ which seems to be an expected ${ }^{2}$ property, i.e. a full ignorant source doesn't impact the fusion result.

The conditioning of a given bba $m($.$) by a conditional$ element $Z \in 2^{\Theta} \backslash\{\emptyset\}$ has been also proposed by Shafer [1]. This function $m(. \mid Z)$ is obtained by DS combination of $m($.$) with the bba m_{Z}($.$) only focused on Z$, i.e. such that $m_{Z}(Z)=1$. For any element $X$ of the power set $2^{\Theta}$ this is mathematically expressed by

$$
m(X \mid Z)=\left[m \oplus m_{Z}\right](X)=\left[m_{Z} \oplus m\right](X)
$$

It has been proved [1] that this rule of conditioning expressed in terms of plausibility functions yields to the formula

$$
P l(X \mid Z)=P l(X \cap Z) / P l(Z)
$$

which is very similar to the well-known Bayes formula $P(X \mid Z)=P(X \cap Z) / P(Z)$. Because of this, DST has been widely considered as a generalization of Bayesian inference [3], or equivalently, that probability theory is a special case of the Mathematical Theory of Evidence when manipulating Bayesian bba's.

Despite of the appealing properties of DS rule, its apparent similarity with Bayes formula for conditioning, and many attempts to justify its foundations, several challenges on the theory's validity have been put forth in the last decades, and remain unanswered. For instance, an experimental protocol to test DST was proposed by Lemmer in 1985 [9], and his analysis shows an inherent paradox (contradiction) of DST. Following a different approach, an inconsistency in the fundamental postulates of DST was proved by Wang in 1994 [11]. Some other related works have been listed in the introduction of this paper. In Section IV, we show through a basic emblematic example where does the problem of DS rule comes from, and why it is very risky to use it in very sensible applications specially where security, defense and safety are involved. Before this, we just recall in the next section the principle of the Proportional Conflict Redistribution rule no. 5 (PCR5) defined within DSmT framework [15] to combine bba's.

\section{BASICS OF PCR5 FUSION RULE}

The idea behind the Proportional Conflict Redistribution rule no. 5 defined within DSmT [15] (Vol. 2) is to transfer conflicting masses (total or partial) proportionally to nonempty sets involved in the model according to all integrity constraints. The general principle of PCR rules is to: 1) calculate the conjunctive consensus between the sources of evidences; 2) calculate the total or partial conflicting masses; 3) redistribute the conflicting mass (total or partial) proportionally on non-empty sets involved in the model according to all integrity constraints. Under Shafer's model assumption ${ }^{3}$ of the frame $\Theta$, the PCR5 combination rule for only two

\footnotetext{
${ }^{2} \mathrm{~A}$ discussion on this topic can be found in [19].

${ }^{3}$ We consider only Shafer's model in this paper and in our examples to make the comparison with Dempster-Shafer's rule results.
} 
sources of information is defined as: $m_{P C R 5}(\emptyset)=0$ and $\forall X \in 2^{\Theta} \backslash\{\emptyset\}$

$$
\begin{aligned}
& m_{P C R 5}(X)=m_{12}(X)+ \\
& \sum_{\substack{Y \in 2^{\Theta} \backslash\{X\} \\
X \cap Y=\emptyset}}\left[\frac{m_{1}(X)^{2} m_{2}(Y)}{m_{1}(X)+m_{2}(Y)}+\frac{m_{2}(X)^{2} m_{1}(Y)}{m_{2}(X)+m_{1}(Y)}\right]
\end{aligned}
$$

All sets involved in the formula (6) are in canonical form. $m_{12}(X)$ corresponds to the conjunctive consensus, i.e:

$$
m_{12}(X)=\sum_{\substack{X_{1}, X_{2} \in 2^{\Theta} \\ X_{1} \cap X_{2}=X}} m_{1}\left(X_{1}\right) m_{2}\left(X_{2}\right) .
$$

All denominators are different from zero. If a denominator is zero, that fraction is discarded. No matter how big or small is the conflicting mass, PCR5 mathematically does a better redistribution of the conflicting mass than DempsterShafer's rule since PCR5 goes backwards on the tracks of the conjunctive rule and redistributes the partial conflicting masses only to the sets involved in the conflict and proportionally to their masses put in the conflict, considering the conjunctive normal form of the partial conflict. PCR5 is quasi-associative and also preserves the neutral impact of the vacuous belief assignment, but contrariwise to DS rule the PCR5 fusion rule doesn't allow the dictatorial power of a source as it will be shown in Section IV. With PCR5 rule, the fusion result can always be revised as soon as informative evidences (i.e. not vacuous bba's) become available.

\section{An EMBlematiC EXAMPLE SHOWING THE DICTATORIAL POWER OF DEMPSTER-SHAFER'S RULE}

Here we present an emblematic example showing the inadequate behavior of Dempster-Shafer's rule. We call this behavior the dictatorial power (DP) of DS rule realized by a given source, which is fundamental in DS reasoning. This parametric example is not related to the level of conflict between sources. In this example the level of conflict can be chosen at any low or high value. We show clearly that Dempster-Shafer's rule is not responding to the combination of different bba's since it provides always one and the same results which is not a good expected behavior for a good fusion rule for applications corresponding to the classical ${ }^{4}$ sense of pooling of evidences.

Let's consider the following frame ${ }^{5} \Theta=\{A, B, C\}$ with Shafer's model. We consider two bba's listed in the Table I, associated with two distinct bodies of evidence ${ }^{6}$ with parameters $a, b_{1}$, and $b_{2}$ that can take any values, as long as $a \in[0,1]$, $b_{1}, b_{2}>0$, and $b_{1}+b_{2} \in[0,1]$.

We grant that all the a priori assumptions below, considered in DST are fulfilled:

1) The sources of evidences are independent;

\footnotetext{
${ }^{4}$ when putting all evidences together.

${ }^{5} \Theta$ could correspond by example to three distinct pathologies of a patient.

${ }^{6}$ In a medical context, the two sources of evidences could correspond to two distinct Doctors providing their own medical diagnostics for a same patient.
}

TABLE I

INPUT BBA's $m_{1}($.$) AND m_{2}($.$) .$

\begin{tabular}{|c|c|c|}
\hline Focal elem. $\backslash$ bba's & $m_{1}()$. & $m_{2}()$. \\
\hline$A$ & $a$ & 0 \\
$A \cup B$ & $1-a$ & $b_{1}$ \\
$C$ & 0 & $1-b_{1}-b_{2}$ \\
$A \cup B \cup C$ & 0 & $b_{2}$ \\
\hline
\end{tabular}

2) Both of sources are equally reliable, i.e both of them are equally truthful. As an additional third assumption in this parametric example we consider:

3 ) Both of sources are truly informative hence no one represents a full ignorant source. It means both sources have their own specific opinions about the particular problem under consideration, which should be taken into account into the fusion process in equal rights manner.

When applying DS rule of combination, one gets:

1) using the conjunctive operator:

$$
\begin{aligned}
m_{12}(A) & =a\left(b_{1}+b_{2}\right) \\
m_{12}(A \cup B) & =(1-a)\left(b_{1}+b_{2}\right) \\
K_{12}=m_{12}(\emptyset) & =1-b_{1}-b_{2} \quad \text { (conflicting mass) }
\end{aligned}
$$

2) after normalizing by $1-K_{12}=b_{1}+b_{2}$, the result is :

$$
\begin{aligned}
m_{D S}(A) & =\frac{m_{12}(A)}{1-K_{12}}=\frac{a\left(b_{1}+b_{2}\right)}{b_{1}+b_{2}} \\
& =a=m_{1}(A) \\
m_{D S}(A \cup B) & =\frac{m_{12}(A \cup B)}{1-K_{12}}=\frac{(1-a)\left(b_{1}+b_{2}\right)}{b_{1}+b_{2}} \\
& =1-a=m_{1}(A \cup B)
\end{aligned}
$$

The final result obtained by using DS rule shows clearly that: - Nevertheless the assumption no. 3 is fulfilled for source $m_{2}$ (.) (it is obviously a truly informative source of evidence), its opinion doesn't count at all in the fusion process, performed by DS rule since one finally gets $m_{D S}()=.m_{1}($.$) . It plays in$ fact a role of full ignorant source, represented by the vacuous belief assignment $m_{v}(A \cup B \cup C)=1$, since $m_{D S}()=.m_{1}($. in the DST fusion process. It is against the required a priori assumption no. 2 of DST, for equally reliable/truthful sources of evidence with opinions that have to be taken into account in equal terms.

- The level of conflict $K_{12}=1-b_{1}-b_{2}$ encountered between the two sources doesn't matter at all in DS fusion process here, since it can be chosen at any level, depending on the choice of $b_{1}+b_{2}$. No matter how high or how low the conflict is, the result remains one and same: $m_{D S}()=.m_{1}($.$) .$

In clear, the source 1 dictates his opinion through DempsterShafer's rule which is what we consider a very inadequate behavior for solving the problem of combination of evidences in practice. Before analyzing this fundamental problem of DST, let's first take the position of devil's advocate, and try to defend the legitimacy of DST's behavior. If we fully trust source 1 , the hypothesis $C$ must be ruled out of the frame, because $\operatorname{Bel}_{1}(C)=P l_{1}(C)=0$. So, according to source 1, the original frame of discernment $\Theta=\{A, B, C\}$ should be 
TABLE II

ADJUSTED INPUT BBA's (STEP 1).

\begin{tabular}{|c|c|c|}
\hline Focal elem. $\backslash$ bba's & $m_{1}()$. & $m_{2}()$. \\
\hline$A$ & $a$ & 0 \\
$A \cup B$ & $1-a$ & $b_{1}$ \\
$C \equiv \emptyset$ & 0 & $1-b_{1}-b_{2}$ \\
$A \cup B \cup \emptyset$ & 0 & $b_{2}$ \\
\hline
\end{tabular}

TABLE III

ADJUSTED INPUT BBA's (STEP 2).

\begin{tabular}{|c|c|c|}
\hline Focal elem. $\backslash$ bba's & $m_{1}()$. & $m_{2}()$. \\
\hline$A$ & $a$ & 0 \\
$A \cup B$ & $1-a$ & $b_{1}+b_{2}$ \\
$C \equiv \emptyset$ & 0 & $1-b_{1}-b_{2}$ \\
\hline
\end{tabular}

reduced to $\Theta^{\prime}=\{A, B\}$, because $C \equiv \emptyset$ (based on the report of source 1). If we consider $C$ impossible to occur, then the report (bba) of source 2 must be adapted/revised according to Tables II and III. Because $m_{2}($.$) must be a normalized$ bba, the masses of all focal elements of $m_{2}($.$) are divided by$ $1-m_{2}(\emptyset)=b_{1}+b_{2}$ so that after adjustment and normalization of $m_{2}($.$) , the two bba's to combine are presented in Table$ IV. Based on this reasoning, we see that the adjusted and normalized bba $m_{2}^{\prime}($.$) plays indeed the role of the vacuous bba$ $m_{v}($.$) when working with the reduced frame \Theta^{\prime}=\{A, B\}$, which perfectly explains the result produced by DS rule.

Such kind of reasoning unfortunately doesn't prove that the result makes sense, nor is it correct. In fact such reasoning shows clearly an asymmetry in the processing, since the source 1 is assumed to provide an absolute certainty on an event " $C$ cannot occur for sure", whereas the source 2 is adjusted (conditioned) by the declaration of source 1. Such devil's advocate reasoning is in fact fallacious, totally mistaken and wrong because it erroneously interprets the impossibility of occurrence of $C$ as a definitive absolute truth (as if all knowledge/evidences were available at the source 1) to withdraw the hypothesis $C$ of the original frame $\Theta$. In fact, the impossibility of $C$ must be interpreted only as conditional truth because it is based only on the partial knowledge related to source 1 (and not on the whole knowledge expressed when pooling the evidences of the two sources).

Let's, just for a comparison purpose, present the respective solution of our example, obtained by DSmT based PCR5 fusion rule. The proportional redistribution of the mass of the partial conflict $m_{1}(A) m_{2}(C)=a\left(1-b_{1}-b_{2}\right)$ is done by

$$
\frac{x_{A}}{m_{1}(A)}=\frac{x_{C}}{m_{2}(C)}=\frac{m_{1}(A) m_{2}(C)}{m_{1}(A)+m_{2}(C)}=\frac{a\left(1-b_{1}-b_{2}\right)}{a+1-b_{1}-b_{2}}
$$

hence $\quad x_{A}=\frac{a^{2}\left(1-b_{1}-b_{2}\right)}{a+1-b_{1}-b_{2}} \quad$ and $\quad x_{C}=\frac{a\left(1-b_{1}-b_{2}\right)^{2}}{a+1-b_{1}-b_{2}}$.

TABLE IV

ADJUSTED BBA's $m_{1}($.$) AND m_{2}^{\prime}($.$) .$

\begin{tabular}{|c|c|c|}
\hline Focal elem. $\backslash$ bba's & $m_{1}()$. & $m_{2}^{\prime}()$. \\
\hline$A$ & $a$ & 0 \\
$A \cup B$ & $1-a$ & $\frac{b_{1}+b_{2}}{1-\left(1-b_{1}-b_{2}\right)}=1$ \\
\hline
\end{tabular}

Similarly the redistribution of the partial conflict mass $m_{1}(A \cup B) m_{2}(C)=(1-a)\left(1-b_{1}-b_{2}\right)$ is done by

$$
\frac{y_{A \cup B}}{m_{1}(A \cup B)}=\frac{y_{C}}{m_{2}(C)}=\frac{m_{1}(A \cup B) m_{2}(C)}{m_{1}(A \cup B)+m_{2}(C)}
$$

hence $y_{A \cup B}=\frac{(1-a)^{2}\left(1-b_{1}-b_{2}\right)}{1-a+1-b_{1}-b_{2}}$ and $y_{C}=\frac{(1-a)\left(1-b_{1}-b_{2}\right)^{2}}{1-a+1-b_{1}-b_{2}}$.

Therefore with PCR5, one gets a fusion result that does react efficiently to the values of all the masses of focal elements of each source since one has:

$$
\begin{aligned}
m_{P C R 5}(A) & =m_{12}(A)+x_{A} \\
& =a\left(b_{1}+b_{2}\right)+\frac{a^{2}\left(1-b_{1}-b_{2}\right)}{a+1-b_{1}-b_{2}} \\
m_{P C R 5}(A \cup B) & =m_{12}(A \cup B)+y_{A \cup B} \\
& =(1-a)\left(b_{1}+b_{2}\right)+\frac{(1-a)^{2}\left(1-b_{1}-b_{2}\right)}{2-a-b_{1}-b_{2}} \\
m_{P C R 5}(C) & =x_{C}+y_{C} \\
& =\frac{a\left(1-b_{1}-b_{2}\right)^{2}}{a+1-b_{1}-b_{2}}+\frac{(1-a)\left(1-b_{1}-b_{2}\right)^{2}}{2-a-b_{1}-b_{2}}
\end{aligned}
$$

In comparison to DS rule performance, the result obtained by using PCR5 rule, shows clearly that PCR5 fusion rule works efficiently in any level of conflict, taking into account all the a priori assumptions $(1-3)$.

\section{Discussion AND ANALYSIS}

The result obtained by DS rule according to the example in Section IV seriously calls in question DS rule's validity, as well as its applicability in real fusion problems. We claim that such a result is not acceptable at all. This example is more crucial than the examples discussed in the existing literature, because it shows clearly a serious flaw in DST behavior, since in this example the level of conflict between sources doesn't play a role, so that it cannot be argued that in such case DS must not be applied because of high conflicting situation. We can choose a low conflict level and the result is still the same. The problem remains and the DST based result could become a source of dramatical consequences, especially in cases, related to human health or security. We claim that the problem behind DS rule behavior comes not from the level of conflict between the sources, but from something else.

\section{A. The dictatorial power of source's minority opinion}

Let's recall again the example, its strange results, and discuss about the reasoning process behind DS rule. The a priori defined finite frame of discernments $\Theta=\{A, B, C\}$ satisfies Shafer's requirement for a set of truly exhaustive and exclusive hypotheses. Lets's first pay attention on the bba associated with source 1 . What is obvious and special from $m_{1}($.$) , it is the fact, that P l_{1}(C)=0$. One can reason from here as follows:

1) Source 1 rules out with absolute certainty the hypothesis $C$ considering it as impossible, because of $P l_{1}(C)=0$. 
TABLE V

INPUT BBA's $m_{1}($.$) AND m_{2}($.$) FOR THE CASE OF TOTAL CONFLICT.$

\begin{tabular}{|c|c|c|}
\hline Focal elem. $\backslash$ bba's & $m_{1}()$. & $m_{2}()$. \\
\hline$A$ & $a$ & 0 \\
$A \cup B$ & $1-a$ & 0 \\
$C$ & 0 & 1 \\
\hline
\end{tabular}

2) The above opinion of source 1 (hypothesis $C$ considered as absolutely impossible) cannot be revised if new informative evidence is available for fusion. According to Shafer's definition [1], $P l_{1}(C)=0$ means for every $X \in 2^{\Theta}$ that $X \cap C \neq \emptyset$, $m_{1}(X)=0$. When DS rule is applied to combine $m_{1}($.$) and$ an arbitrary $m^{\prime}($.$) (in our example m^{\prime}()=.m_{2}($.$) ), for every$ $Y \in 2^{\Theta}$ that $Y \cap C \neq \emptyset, m_{D S}(Y)=0$, because it is the sum of some products, each of them take one of the above $m_{1}(X)$ as a factor. Consequently, $P l_{D S}(C)=0$, no matter what the other source of evidence is.

3) Since with DS rule, the source 1 imposes its own opinion on source 2, and in fact on any other sources (as soon as they have a core including the core of source 1), DST supports the dictatorial power of a given source by accepting the minority opinion as a valid solution of the "fusion of evidences", and by banning in the same time all other sources' different opinions. This behavior is in full contradiction with the a priori assumption no. 2 of DST for equally reliable sources of information, which means their opinions should be taken into account on equal terms in the fusion process.

\section{B. On the total conflict case banned by DST}

Let's try to reveal now what is the logic behind the case, that DS rule cannot solve because of the indefiniteness $(0 / 0)$ - the case of total conflicting sources of information. We consider the same frame of discernments $\Theta=\{A, B, C\}$ and two bba's (listed in a Table V), associated with two distinct bodies of evidence $m_{1}($.$) and m_{2}($.$) with parameter a \in[0,1]$. It is obvious from Table $\mathrm{V}$ that:

1) Source 1 rules out with an absolute certainty hypothesis $C$ considering it as impossible since $P l_{1}(C)=0$.

2) Source 2 rules out with an absolute certainty the hypotheses $A$ and $B$ considering them as impossible since $P l_{2}(A \cup B)=0$.

The a priori DST assumptions $(1-3)$ still hold. So, the question is: Which source will possess the dictatorial power in this special case? Following Shafer's interpretation in this example, the answer is: both of sources have access to the Absolute truth. But what is paradoxical and contradictory is that having simultaneously an access to the Absolute truth, both of sources ban mutually each other opinions.

Therefore Shafer's interpretation that allows both sources to rule out all possible Absolute truths in absolute manner leads to the strong contradiction by accepting the assertion that DS rule cannot be used in such totally conflicting case.

This assertion is substantiated on the obtained mathematical indefiniteness $(0 / 0)$ as impossible "fusion result". But actually behind the formal mathematical explanation, there resides a real and strong logic that Shafer's distinct Absolute truth interpretation granted to each source doesn't hold. The Absolute truth is unique and it cannot yield to contradictions in the fusion process.

For a comparison purpose, let's again to present the respective solution in this special conflicting case, obtained by DSmT based PCR5 fusion rule. The proportional redistribution of the mass of the partial conflict $m_{1}(A) m_{2}(C)=a$ is done by

$$
\frac{x_{A}}{m_{1}(A)}=\frac{x_{C}}{m_{2}(C)}=\frac{m_{1}(A) m_{2}(C)}{m_{1}(A)+m_{2}(C)}=\frac{a}{1+a}
$$

with $\quad x_{A}=\frac{a^{2}}{1+a} \quad$ and $\quad x_{C}=\frac{a}{1+a}$.

Similarly the proportional redistribution of the partial conflict mass $m_{1}(A \cup B) m_{2}(C)=(1-a)$ is done by

$$
\frac{y_{A \cup B}}{m_{1}(A \cup B)}=\frac{y_{C}}{m_{2}(C)}=\frac{m_{1}(A \cup B) m_{2}(C)}{m_{1}(A \cup B)+m_{2}(C)}
$$

with $\quad y_{A \cup B}=\frac{(1-a)^{2}}{2-a} \quad$ and $\quad y_{C}=\frac{(1-a)}{2-a}$.

Finally, one gets using PCR5 fusion rule

$$
\begin{aligned}
m_{P C R 5}(A) & =x_{A}=\frac{a^{2}}{1+a} \\
m_{P C R 5}(A \cup B) & =y_{A \cup B}=\frac{(1-a)^{2}}{2-a} \\
m_{P C R 5}(C) & =x_{C}+y_{C}=\frac{a}{1+a}+\frac{(1-a)}{2-a}
\end{aligned}
$$

It is obvious, DSmT based PCR5 fusion rule works efficiently even in this special total conflicting case. This very attractive rule is just a non-Bayesian reasoning approach, which is not based on such inherent contradiction, as DST, because PCR5 doesn't support Shafer's interpretation of source committed Absolute truth and doesn't allow dictatorial power of single source opinion on all other sources, involved in the fusion.

\section{Remark on Dempster-Shafer conditioning}

Some comments must be given also about DS conditioning rule (4) and the expression (5) for the conditional plausibility. Let consider $\Theta$ and two bba's $m_{1}($.$) and m_{2}($.$) defined on$ $2^{\Theta}$ and their DS combination $m_{D S}()=.\left[m_{1} \oplus m_{2}\right]($.$) and$ let assume a conditioning element $Z \neq \emptyset$ in $2^{\Theta}$ and the bba $m_{Z}(Z)=1$, then

$$
m_{D S}(. \mid Z)=\left[m_{D S} \oplus m_{Z}\right](.)=\left[m_{1} \oplus m_{2} \oplus m_{Z}\right](.)
$$

Because $m_{D S}()=.\left[m_{1} \oplus m_{2}\right]($.$) is inconsistent with the$ probability calculus [10], [11], [13], [14], [19], then $m_{D S}(. \mid Z)$ is also inconsistent. Therefore for any $X$ in $2^{\Theta}$, the conditional plausibility $P l(X \mid Z)$ expressed by $P l(X \mid Z)=P l(X \cap$ $Z) / P l(Z)$ obtained from $m_{D S}(. \mid Z)$, having an apparent similarity with Bayes formula, is in fact not compatible with the conditional probability as soon as several sources of evidences are involved. 


\section{FUNDAMENTAL THEOREM ON THE INHERENT CONTRADICTION IN DST FOUNDATIONS}

On the base of the previous examples and after a detailed analysis of results drawn from Dempster-Shafer's rule and DST reasoning discussed in previous section, we establish the fundamental theorem on the inherent contradiction of DST foundations.

Theorem : Dempster-Shafer Theory is wrong because its foundation is based on an inherent logical contradiction.

Proof : In the basis of DST [1], Shafer considers:

- An a priori defined finite frame of discernment $\Theta=$ $\left\{\theta_{1}, \theta_{2}, \ldots, \theta_{n}\right\}$ with $n \geq 2$, satisfying Shafer's requirement for a set of truly exhaustive and exclusive hypotheses. Recalling Shafer's statement about DST [1] (p. 36): "This formalism is most easily introduced in the case where we are concerned with the true value of some quantity. If we denote the quantity by $\theta$ and the set of its possible values by $\Theta$, then the propositions of interest are precisely those of the form "The true value of $\theta$ is in $T$," where $T$ is a subset of $\Theta$ ".

- Available independent sources of evidences associated with corresponding bba's $m_{i}(),. i=1,2 .$. , where all the sources are equally reliable/trustable and can be truly informative (not fully ignorant).

- The level of conflict between the sources can take any low or high value strictly less than one to make Dempster-Shafer's rule mathematically defined.

On the base of above considerations, one encounters the fundamental contradiction:

1) A given source of evidence $m_{p}($.$) can become unrevis-$ able during the fusion when it is allowed to rule out with absolute certainty some hypothesis $\theta_{k}, k \in[1, n]$ in the frame $\Theta$ (if $P l_{p}\left(\theta_{k}\right)=0$ as shown in our emblematic example).

2) DS rule cannot solve the case of total conflict between the sources (because of mathematical indefiniteness $0 / 0)$. This corresponds to the case when both sources: 1) have an access to the Absolute truth;2) can become unrevisable during the fusion if they allowed to rule out with absolute certainty all hypotheses in the frame $\Theta$, banning mutually each other opinions. The inability of DS rule to solve this case strongly supports the assertion that the Absolute truth must be unique. Otherwise the total conflict case could also be solved/processed by DS rule. So, Shafer's interpretation of distinct Absolute truth granted to each source does not hold.

Therefore from the point 2), DST agrees with the assertion that the Absolute truth is unique and cannot be a contradiction. This assertion is fully contradicting with Shafer's interpretation of distinct Absolute truth granted to each source stated in point 1). This proves the fundamental contradiction in the foundations of DST and completes the proof of our Theorem.

\section{CONCLUSION}

In this paper, we have identified and put in light the very serious inherent contradiction of Dempster-Shafer Theory foundations. On the base of simple emblematic example, we have analyzed and explained the inconsistent and inadequate behavior of Dempster-Shafer's rule of combination as a valid method for the combination of sources of evidences. We have identified the cause and the effect of the dictatorial power behavior of this rule and of its impossibility to manage the conflicts between the sources. For a comparison purpose, the respective solutions obtained by the more adequate PCR5 fusion rule, proposed originally in Dezert-Smarandache Theory framework, were presented. This very attractive rule corresponds to a non-Bayesian reasoning approach, which is not based on such inherent contradiction, as DST, because PCR5 doesn't support Shafer's interpretation of source committed Absolute truth and doesn't allow dictatorial power of single source opinion on all other sources, involved in the fusion.

\section{REFERENCES}

[1] Shafer, G. A Mathematical Theory of Evidence. Princeton University Press, Princeton, 1976.

[2] Dempster, A. Upper and lower probabilities induced by a multivalued mapping, Ann. Math. Statist., Vol. 38, 1967, pp. 325-339.

[3] Dempster, A. A generalization of Bayesian inference, J. R. Stat. Soc. B 30, 1968, pp. 205-247.

[4] Dempster, A. The Dempster-Shafer calculus for statisticians, IJAR, Vol. 48, 2008, pp. 365-377.

[5] Smets, P. Practical uses of belief functions. in K. B. Lskey and H. Prade, Editors, UAI 99, Stockholm, Sweden, 1999, pp. 612-621.

[6] Zadeh, L.A. On the validity of Dempster's rule of combination, Memo M79/24, Univ. of California, Berkeley, U.S.A., 1979.

[7] Zadeh, L.A. Book review: A mathematical theory of evidence, The Al Magazine, Vol. 5 (3), 1984, pp. 81-83.

[8] Zadeh, L.A. A simple view of the Dempster-Shafer theory of evidence and its implication for the rule of combination, The Al Magazine, Vol. 7 (2), 1986, pp. 85-90.

[9] Lemmer, J. Confidence factors, empiricism and the Dempster-Shafer theory of evidence, in Proc. of 1st Conf. on Uncertainty in Artificial Intelligence (UAI-85), 1985, pp. 160-176.

[10] Voorbraak, F. On the justification of Demspster's rule of combination, Dept. of Philosophy, Univ. of Utrecht, The Netherlands, Logic Group Preprint Series, No. 42, Dec. 1988.

[11] Wang, P. A defect in Dempster-Shafer theory, in Proc. of 10th Conf. on Uncertainty in AI, 1994, pp. 560-566.

[12] Pearl, J. Reasoning with belief functions: An analysis of compatibility, Int. Journal of Approximate Reasoning, Vol. 4, 1990, pp. 363-389.

[13] Walley, P. Statistical Reasoning with Imprecise Probabilities, Chapman and Hall, London, 1991, pp. 278-281.

[14] Gelman, A. The boxer, the wrestler, and the coin flip: a paradox of robust Bayesian inference and belief functions, American Statistician, Vol. 60, No. 2, 2006, pp. 146-150.

[15] Smarandache F., Dezert J. Advances and applications of DSmT for information fusion, Volumes 1, 2 \& 3, ARP, 2004-2009.

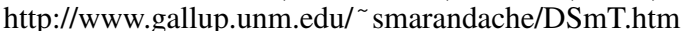

[16] Smets P., Kennes R. The transferable belief model, Artif. Int., Vol. 66 , 1994, pp. 191-234.

[17] Smets, P. The transferable belief model for quantified belief representation, Handbook of Defeasible Reasoning and Uncertainty Management Systems, Vol.1, Kluwer, 1998.

[18] Dezert J., Tchamova A. On the behavior of Dempster's rule of combination, Presented at the spring school on Belief Functions Theory and Applications (BFTA), Autrans, France, 4-8 April 2011 (http://hal archives-ouvertes.fr/hal-00577983/).

[19] Dezert J., Tchamova A., Dambreville F. On the mathematical theory of evidence and Dempster's rule of combination, May 2011(http://hal. archives-ouvertes.fr/hal-00591633/fr/). 